\title{
I NUMERI DEGLI IMMIGRATI
}

\author{
CARLA GE RONDI (*)
}

Nota presentata dal m.e. Silvio Beretta

(Adunanza del 26 novembre 2015)

SuNTO. - Il lavoro affronta sia il problema di definire il fenomeno migratorio sia quello di pervenire a stime di esso comparabili nel tempo e nello spazio. Una prima parte è quindi dedicata alle definizioni adottate nei sistemi statistici cui più di consueto si fa riferimento (ISTAT, ONU, IOM, WORLD BANK, EUROSTAT). La seconda parte prende in esame criticamente i dati resi disponibili dall'ISTAT per quanto riguarda l'Italia, da EUROSTAT per quanto riguarda i paesi dell'Unione Europea e, infine, dall'ONU per quanto riguarda i paesi del mondo. I dati delle migrazioni internazionali desunti da fonte ONU sono presi in esame tenendo distinti quelli che si riferiscono a paesi che adottano come definizione di "immigrato" (migrant) il criterio della cittadinanza, dai paesi che adottano come definizione di "immigrato" (migrant) il criterio della nascita. Infine, con riferimento all'Italia, viene proposto un confronto tra i dati relativi ai migranti (secondo il paese di nascita) desunti dall'ONU e gli immigrati stranieri (secondo il paese di cittadinanza) desunti dall'ISTAT.

$$
* * *
$$

ABSTRACT. - After acknowledging the existence of different definitions of migrant, this work addresses the issue of achieving estimates that are comparable across time and space. Consequently, the first part of the work focusses on the definitions of migrant adopted by widely used statistical sources of migration data (ISTAT, UN, IOM, WORLD BANK, EUROSTAT). The second part critically analyses the migration data provided by ISTAT, EUROSTAT and UN, respectively referring to Italy, Europe and the world. As regards UN data on international migrations, the countries that define migrants by citizenship were kept separate from those that define them by place of birth. Finally, a comparison is offered between the data on immigration in Italy provided by UN (defined by place of birth) and those provided by ISTAT (defined by citizenship). 


\section{INTRODUZIONE}

\section{Negli ormai lontani anni Sessanta scriveva Nora Federici':}

Non è facile dare una definizione esatta ed univoca di migrazione poiché il concetto comune è di per sé stesso abbastanza vago e sta generalmente ad indicare lo spostamento di sede. Dal punto di vista statistico, tuttavia, sarebbe necessario precisare il significato del termine non solo perché tale precisazione deve costituire la base per la rilevazione e la misura del movimento migratorio, ma anche perché soltanto se questo viene chiaramente definito si possono valutare correttamente le conseguenze demografiche, economiche e sociali delle migrazioni.

In effetti, però, anche la definizione statistica si presenta tutt'altro che facile in quanto la realtà economico-sociale nella quale il fenomeno migratorio si attua è estremamente mutevole e dà a questo caratteristiche e portata di volta in volta diverse. Di conseguenza i criteri cui si attengono i vari Paesi per definire i migranti non sono gli stessi ed anche in uno stesso Paese tali criteri mutano nel tempo. Come criterio oggettivo per isolare, nell'ambito degli spostamenti territoriali, le migrazioni propriamente dette si può assumere la durata oppure il motivo dello spostamento.

\section{La Federici rilevava quindi che}

la eventuale confusione tra i due criteri della durata e del motivo toglie alla classificazione un significato preciso e crea, inoltre, condizioni sostanziali di non comparabilità dei dati ${ }^{2}$

e concludeva, quindi, che dovrebbe venire attribuita la qualifica di migrazioni ai soli spostamenti determinati dalla pressione demografica differenziale intendendo per tale il rapporto tra popolazione e risorse oppure il rapporto tra tasso annuo di incremento naturale e tasso annuo di sviluppo economico (ad esempio di incremento del reddito $)^{3}$.

Si è ritenuto di dare spazio a questa lunga citazione poiché, nel

1 N. Federici, Lezioni di Demografia, Roma, Editrice E. De Santis, 1968, 388.

2 N. Federici, Lezioni di Demografia, cit., 389.

3 Attualmente chiameremmo in causa la differenza nel livello di sviluppo umano. 
corso del mezzo secolo che è intercorso da quando è stata scritta, la difficoltà di definire il fenomeno migratorio non è minimamente scemata, come è rimasta più che mai attuale quella di pervenire a stime di esso comparabili nel tempo e nello spazio. In questo mezzo secolo, d'altro canto, si sono sostanzialmente modificate le modalità con cui il fenomeno si presenta all'attenzione non solo degli studiosi ma altresì a quella dei decisori pubblici fino ai mezzi di comunicazione.

E' quindi d'obbligo sottolineare che quando la Federici trattava di "migrazione" aveva per lo più presente la componente "emigrazione" 4 , quella che l'Italia stava sperimentando dalla seconda metà del XIX secolo, ben rari essendo i casi di immigrazione se non di cittadini italiani che facevano ritorno al loro Paese.

Per poter effettuare confronti nel tempo e nello spazio occorre, come già è stato sottolineato, condividere un linguaggio comune e comuni criteri di rilevazione e di stima. E a proposito di rilevazione e di stima è di fondamentale importanza distinguere se si stanno analizzando dati di flusso oppure di stock, essendo peraltro chiaro che se "immigrazione" denota un processo, e quindi si configura come un flusso, "immigrato" denota un ammontare e, quindi, è un dato di stock. Qui di seguito si elencheranno, pertanto, le definizioni del fenomeno adottate nei sistemi statistici cui più di consueto si fa riferimento.

\section{LE FONTI}

1) Nel Glossario dell'ISTAT (Istituto Nazionale di Statistica) i termini "immigrato" e "immigrazione" non sono presenti. Sono invece definite le "iscrizioni per trasferimento di residenza dall'estero" avvenute durante un certo arco temporale (un anno, per esempio) e che possono riguardare, appunto, sia gli italiani (in tale caso si parla di "rimpatri") sia i cittadini stranieri che, a seguito di una immigrazione, hanno assunto la residenza in un comune del nostro Paese e che risultano quindi residenti ("cittadini stranieri residenti") ad una

4 Lo spostamento di popolazione viene denominato emigrazione quando ci si riferisce alla sede di provenienza dalla quale l'individuo si allontana e immigrazione quando ci si riferisce alla sede di destinazione nella quale un individuo si stabilisce: $\mathrm{cfr}$. N. Federici, Lezioni di Demografia, cit., 391. 
determinata data (di solito il 31 dicembre di ogni anno) $)^{5}$. Si deve sottolineare che, dunque, "immigrato" e "straniero" non sono affatto sinonimi.

2) L'ONU definisce e stima l'International migrant stock in questi termini:

international migrants have been equated with the foreign-born population whenever this information is available, which is the case in most countries or areas. In most countries lacking data on place of birth, information on the country of citizenship of those enumerated was available, and was used as the basis for the identification of international migrants, thus effectively equating, in these cases, international migrants with foreign citizens ${ }^{6,7}$.

Della definizione ONU si deve necessariamente sottolineare che migrant è considerato sia chi è nato in un paese diverso sia chi non ha la cittadinanza del paese in cui vive. Tale duplice e ambigua definizione influisce pertanto sulle statistiche del fenomeno a livello internazionale, producendo stime che possono essere discordanti benché non sia possibile apprezzare in termini quantitativi tale discordanza. Più organismi statistici internazionali si rifanno alla definizione ONU sopra riportata ma, come si vedrà, acquisendola non integralmente nei rispettivi glossari. Eccone alcuni esempi.

3) L'IOM (International Organization for Migration) pone l'attenzione sul termine migrant precisando che

At the international level, no universally accepted definition for 'migrant' exists. The term migrant was usually understood to cover all cases where the decision to migrate was taken freely by the individual concerned for reasons of 'personal convenience' and without intervention of an external compelling factor; it therefore applied to persons,

5 http://www3 istat.it/cgi-bin/glossario/indice.pl. Può ancora essere utile ricordare che nelle Serie storiche dell'ISTAT sono riportate sia le statistiche degli espatriati e dei rimpatriati dall'ultimo trentennio del XIX secolo fino al primo quinquennio del XXI secolo, sia le statistiche degli stranieri residenti dal 1981 al 2009 ( http://seriestoriche.istat.it/).

6 http://www.un.org/en/development/desa/population/migration/data/estimates2/docs/MigrationStockDocumentation 2015.pdf

7 http://www.un.org/en/development/desa/population/migration/data/estimates2/docs/MigrationStockDocumentation_2015.pdf 
and family members, moving to another country or region to better their material or social conditions and improve the prospect for themselves or their family. The United Nations defines migrant as an individual who has resided in a foreign country for more than one year irrespective of the causes, voluntary or involuntary, and the means, regular or irregular, used to migrate. Under such a definition, those travelling for shorter periods as tourists and businesspersons would not be considered migrants. However, common usage includes certain kinds of shorter-term migrants, such as seasonal farm-workers who travel for short periods to work planting or harvesting farm products.

In sintesi, definisce migrant

as any person who is moving or has moved across an international border or within a State away from his/her habitual place of residence, regardless of (1) the person's legal status; (2) whether the movement is voluntary or involuntary; (3) what the causes for the movement are; or (4) what the length of the stay is ${ }^{8}$.

Si deve sottolineare che la definizione data all'IOM è più articolata di quella ONU chiamando in causa, in particolare, anche il motivo che ha determinato la migrazione: eppure, come facilmente si potrebbe verificare, i dati che tale Organizzazione propone sono precisamente uguali a quelli di fonte $\mathrm{ONU}^{9}$.

\section{4) Per la World BAnK:}

International migrant stock is the number of people born in a country other than that in which they live. It also includes refugees. The data used to estimate the international migrant stock at a particular time are obtained mainly from population censuses. The estimates are derived from the data on foreign-born population, people who have residence in one country but were born in another country. When data on the foreign-born population are not available, data on foreign population - that is, people

\section{8 http://www.iom.int/key-migration-terms}

9 http://www.iom.int/world-migration. A titolo di esempio riportiamo che da tale fonte si desume che nel 2015 gli immigrati in Italia erano 5.788 .875 ossia il 9.68\% della popolazione residente: è il dato fornito, appunto, dall'ONU che fa riferimento ai residenti nati all'estero e che, come si vedrà, differisce da quello di fonte ISTAT che fa riferimento agli immigrati stranieri. 
who are citizens of a country other than the country in which they reside - are used as estimates ${ }^{10}$.

5) EUROstaT propone un'articolato glossario sul fenomeno migratorio. Definisce innanzitutto lo straniero

Foreign population or foreigners refer to persons who are not citizens of the country in which they reside, including persons of unknown citizenship and stateless persons ${ }^{11}$.

Precisa poi che

"Migration refers to the number of migrants, people changing their residence to or from a given area (usually a country) during a given time period (usually one year)". "Immigrants are people arriving or returning from abroad to take up residence in a country for a certain period, having previously been resident elsewhere. According to the 1998 United Nations recommendations on the statistics of international migration (Revision 1), an individual is a long-term immigrant if he/she stays in his/her country of destination for a period of 12 months or more, having previously been resident elsewhere for 12 months or more". "Emigrants are people leaving the country where they usually reside and effectively taking up residence in another country. According to the $1998 \mathrm{UN}$ recommendations on the statistics of international migration (Revision 1), an individual is a long-term emigrant if he/she leaves his/her country of previous usual residence for a period of 12 months or more" ${ }^{12}$.

Si è ritenuto di riportare le diverse definizioni di "migrante" ovvero di "immigrato" adottate in sede internazionale in quanto esiste un legame molto stretto tra disponibilità di informazioni statistiche ed analisi del fenomeno: la seconda, senza le prime, non sarebbe in alcun modo attuabile, in quanto non documentabile. Si deve inoltre sottolineare, in merito, che le informazioni statistiche rese disponibili sono prodotte dal paese di arrivo poiché è questo stesso che ha interesse a

10 http://data.worldbank.org/indicator/SM.POP.TOTL

11 http://ec.europa.eu/eurostat/statistics-explained/index.php/Glossary: Foreign_population

12 http://ec.europa.eu/eurostat/statistics-explained/index.php/Glossary: Migrant 
rilevare quanti stranieri si trovano sul suo territorio mentre, salvo casi eccezionali, il paese di origine non ha interesse a rilevare quanti lo abbandonano senza contare che, nella assoluta maggioranza dei casi, i paesi che generano un flusso emigratorio sono gli stessi in cui le condizioni sociali, ambientali e politiche impediscono di fatto la corretta ed esaustiva rilevazione di qualsiasi evento demografico e, a maggior ragione, se questi eventi si riferiscono al trasferimento di persone da un territorio ad un altro. Nella norma, dunque, il controllo è effettuato tramite procedure amministrative, come il rilascio di visti o permessi di soggiorno o di lavoro, solo sulle persone che entrano in un dato paese.

Nel seguito si utilizzeranno come statistiche di riferimento quelle rese disponibili dall'ONU in quanto l'impiego di un'unica fonte consente con ragionevole affidabilità di confrontare i dati nello spazio e nel tempo. L'ONU fornisce, infatti, le statistiche relative all'International Migrant stock secondo il paese di origine e di destinazione per intervalli quinquennali a partire dal 1990 fino al 2015. Le stime, poiché di stime si tratta e questo va sottolineato (non è incauto ipotizzare che eventuali errori o distorsioni possano essere di tipo sistematico e, quindi, influire univocamente), sono riferite al 1 luglio. Per ogni paese è specificato

whether the data used to produce the estimates refer to the foreign-born population (B) or to foreign citizens (C). It also indicates in which cases the number of refugees, as reported by UNHCR, were added to the estimate of international migrants (R). Estimates for countries or areas having no data on the number of international migrants were obtained by imputation indicated by $(\mathrm{I})^{13}$.

\section{I DATI}

Dalla pluralità delle definizioni adottate in sede internazionale dei "migranti" ovvero "immigrati" e, conseguentemente, dalla non univoca identificazione del loro paese di provenienza, considerato talvolta quello di nascita, talvolta quello di cittadinanza e talvolta ancora l'uno o l'altro a seconda delle informazioni disponibili, non può che scaturire una plu-

13 United Nations, Department of Economic and Social Affairs (2015). Trends in International Migrant Stock: Migrants by Destination and Origin (United Nations database, POP/DB/MIG/Stock/Rev.2015). 
ralità di stime della consistenza (stock) del fenomeno anche molto difformi tra loro.

Conviene, come è stato fatto per le definizioni, prendere in considerazione in primo luogo i dati dell'Italia ribadendo che si porrà l'attenzione solo sugli stranieri. La consistenza degli stranieri immigrati è rilevata dall'ISTAT o in sede censuaria con cadenza decennale, oppure attraverso le registrazioni anagrafiche con cadenza mensile o annuale. Attraverso il censimento è possibile conoscere la distribuzione degli stranieri sia per cittadinanza sia per luogo di nascita; le registrazioni anagrafiche rendono nota solo la distribuzione degli stranieri per cittadinanza.

Secondo il Censimento della Popolazione effettuato nel 2011, dei 4.027.627 stranieri censiti l'85 per cento era nato all'estero mentre i rimanenti 608.623 erano stranieri nati in Italia: gli immigrati, ossia gli stranieri nati all'estero, sarebbero pari a 3.419.004 unità. Gli stranieri nati in Italia non sono immigrati, è evidente, mentre tali sarebbero considerati gli italiani nati all'estero e poi entrati nel paese ${ }^{14}$.

Le statistiche rese disponibili da EUROSTAT consentono corretti confronti tra $\mathrm{i}$ paesi che fanno parte dell'Unione ${ }^{15}$. Precisa infatti EUROSTAT che

Dal 2008 la rilevazione dei dati in materia di migrazioni, cittadinanza e asilo si basa sulle disposizioni del regolamento (CE) n. 862/2007 (si veda); l'analisi e la composizione dei gruppi di paesi dell'UE, dell'EFTA e dei paesi candidati all'adesione al $1^{\circ}$ gennaio dell'anno di riferimento sono riportate nel regolamento di esecuzione (UE) $\mathrm{n}$. 351/2010, che definisce una serie di statistiche di base sui flussi migra-

14 Per quanto è chiara e priva di ambiguità ci sembra utile richiamare qui la definizione di "immigrato" utilizzata in Francia dall'INSEE: "Selon la définition adoptée par le Haut Conseil à l'Intégration, un immigré est une personne née étrangère à l'étranger et résidant en France. Les personnes nées françaises à l'étranger et vivant en France ne sont donc pas comptabilisées. À l'inverse, certains immigrés ont pu devenir français, les autres restant étrangers. Les populations étrangère et immigrée ne se confondent pas totalement: un immigré n'est pas nécessairement étranger et réciproquement, certains étrangers sont nés en France (essentiellement des mineurs). La qualité d'immigré est permanente: un individu continue à appartenir à la population immigrée même s'il devient français par acquisition. C'est le pays de naissance, et non la nationalité à la naissance, qui définit l'origine géographique d'un immigré".

15 http://ec.europa.eu/eurostat/statistics-explained/index.php/Migration_and_ migrant_population_statistics 
tori internazionali, sugli stock di popolazione straniera, sulle acquisizioni di cittadinanza e sui permessi di soggiorno, nonché in materia di asilo e di misure contro l'ingresso e il soggiorno illegali. Sebbene gli Stati membri dell'UE possano continuare a utilizzare altri dati, purché affidabili, in funzione della loro disponibilità e delle prassi nazionali, le statistiche compilate a norma del regolamento devono basarsi su definizioni e concetti comuni.

EUROSTAT rende disponibili tavole con l'ammontare degli stranieri, paese per paese, secondo la cittadinanza (cittadini di un altro Paese comunitario, cittadini non comunitari) e degli individui nati all'estero per paese di nascita (altro paese dell'Unione, paese non appartenenti all'Unione). Merita riflettere in proposito che:

a) gli Stranieri sono costituiti sia da coloro che sono nati nel paese sia da coloro che sono nati in un altro paese $\left(S_{\text {tot }}=S_{\text {natiIn }}+S_{\text {natiOut }}\right)$;

b) i Nati all'estero sono costituiti sia dai cittadini nati all'estero sia dagli stranieri nati all'estero $\left(\mathrm{N}_{\text {out }}=\mathrm{C}_{\text {natiOut }}+\mathrm{S}_{\text {natiOut }}\right)$.

Ne consegue che la differenza tra Stranieri e Nati all'estero è uguale alla differenza tra gli stranieri nati nel Paese e i cittadini nati fuori $\left(\mathrm{S}_{\text {tot }}-\mathrm{N}_{\text {out }}=\mathrm{S}_{\text {natiIn }}-\mathrm{C}_{\text {natiOut }}\right)$. La differenza sarà quindi positiva se la consistenza degli stranieri nati nel paese è superiore a quella dei cittadini nati all'estero, negativa nel caso opposto. Trattandosi di un saldo non è ovviamente possibile stimare la consistenza delle due componenti.

La Tab. 1 presenta, per i paesi più interessati dal fenomeno immigratorio, la distribuzione degli stranieri per cittadinanza e quella degli individui nati all'estero per paese di nascita, al 1 gennaio 2014.

La Germania è il paese che conta la più elevata presenza sia di stranieri ( 7 milioni di cui oltre la metà, ossia il 56 per cento, cittadino di un paese extracomunitario) sia di nati all'estero ( 9,8 milioni il 60 per cento dei quali nato in un paese al di fuori della UE) ma è il Lussemburgo il paese che conta tra la popolazione la maggiore percentuale sia di stranieri sia di nati all'estero. La Grecia e l'Italia contano la più elevata presenza di cittadini di paesi extracomunitari (oltre il 70 per cento) mentre Lussemburgo, Belgio e Irlanda sono i paesi che ne ospitano nella percentuale minore (14 per cento il Lussemburgo e poco più del 30 per cento Belgio e Irlanda). La Grecia è ancora il Paese in cui è relativamente più frequente la presenza di nati fuori dall'area comuni- 
taria ( 73 per cento), seguita dalla Francia ( 71 per cento). I nati in un paese che non appartiene all'Unione sono minimamente frequenti ancora in Lussemburgo e poi in Irlanda. E' d'obbligo constatare che, comunque la si voglia definire, l'immigrazione in Lussemburgo interessa i paesi dell'Unione mentre quella in Grecia interessa gli altri paesi. Il Lussemburgo, che si configura pertanto come un paese popolato da immigrati prevalentemente di origine europea, è altresì l'unico, tra quelli qui presi in considerazione, che presenta un saldo positivo a favore degli stranieri: gli stranieri nati nel paese sono quindi più numerosi dei lussemburghesi nati all'estero. L'Italia, dei 13 considerati, è il paese che conta tra la propria popolazione la minore presenza relativa di cittadini nati all'estero (9,4 per cento dei residenti) mentre i Paesi Bassi sono il paese che conta la minore percentuale di stranieri $(4,4$ per cento della popolazione). Un'altra peculiarità dell'Olanda che non si può non rilevare è che gli olandesi nati all'estero superano del 165\% gli stranieri nati in Olanda.

Infine vale la pena di sottolineare che, se si ordinano i 13 paesi secondo la percentuale crescente sia degli stranieri sia dei nati all'estero e si confrontano tra di loro i due ordinamenti mediante, per esempio, il coefficiente di correlazione di rango ${ }^{16}$, si conclude che fra di essi vi è una forte relazione diretta (il coefficiente presenta un valore pari a 0,72 , sapendo che 1 è il suo valore massimo). In altre parole, questo consente di ritenere che la stima della frequenza dei nati all'estero sia accettabile come stima di quella della presenza straniera e viceversa, sempre che non si tenga conto, evidentemente, delle diversità delle rispettive consistenze. D'altra parte, se consideriamo "immigrati" coloro che sono nati all'estero è sicuramente interessante chiedersi quali siano i loro principali paesi di origine in alcuni degli Stati dell'UE sulla base dei dati resi disponibili da EUROSTAT.

Come si desume dalla Tab. 2 che, con riferimento al 1 gennaio 2014 , prende in considerazione i principali paesi di immigrazione ${ }^{17}$, tra i paesi di origine prevale la Romania (merita citare che in Romania i nati in Italia sono quasi 26 mila) seguita da Albania, Marocco, Ucraina

16 Si veda, in proposito, per esempio: L. Muttarini, Metodi statistici applicati alle ricerche economiche e sociali, Milano, Giuffrè, 1974, 314-316.

17 Il dato non è disponibile per Francia, Grecia, Germania, Croazia, Cipro, Lussemburgo, Malta, Austria, Polonia. 
e Germania. Il Marocco è fra i più frequenti paesi di origine anche degli immigrati in Belgio, Paesi Bassi e Spagna. Numerosi sono in Spagna coloro che sono nati in America Latina: 429 mila gli equadoregni e 353 mila i colombiani. La Svezia più degli altri Stati conta immigrati originari di Iraq (quasi 129 mila) e Iran (67 mila). Nel Regno Unito sono relativamente più numerosi i nati in India (772 mila) e Pakistan (524 mila). I turchi di nascita sono più che altrove numerosi in Belgio e soprattutto nei Paesi Bassi dove si presentano primi in graduatoria. Merita citare infine che nati in Somalia sono presenti in Finlandia e in Norvegia.

Se si vuole estendere l'orizzonte investigativo dal sistema UE al sistema mondiale, occorre fare riferimento alle statistiche ONU, che forniscono la distribuzione dello stock di migranti secondo l'origine e la destinazione per intervalli quinquennali dal 1990 al 2015 $5^{18}$. Occorre sottolineare in proposito che questi dati vengono mutuati da parte di altri organismi internazionali come l'International Migration Organization che, come si è rilevato più sopra, pure adottano definizioni dello stock di migranti più articolate di quelle ONU.

Prima di descrivere, necessariamente in forma sommaria, come si presenta ora (2015) la migrazione internazionale e come si è presentata nel passato, precisiamo che dei 232 paesi di destinazione presi in considerazione nelle tavole ONU, 180 tengono in conto come paese di origine quello di nascita e 46 quello di cittadinanza. Per i 6 paesi che non hanno fornito statistiche in merito (Eritrea, Somalia, Sahara Occidentale, Repubblica democratica di Corea, Bosnia e Erzegovina, Santa Sede), l'ONU ha stimato a calcolo il numero degli immigrati. In taluni casi le stime comprendono i rifugiati come definiti dall'UNHCR ${ }^{19}$ :

Refugees include individuals recognised under the 1951 Convention relating to the Status of Refugees; its 1967 Protocol; the 1969 OAU Convention Governing the Specific Aspects of Refugee Problems in Africa; those recognised in accordance with the UNHCR Statute; indi-

18 Per il procedimento di stima si veda: United Nations, Department of Economic and Social Affairs, Trends in International Migrant Stock: The 2015 Revision (CD-ROM Documentation) e http://www.un.org/en/development/desa/population /migration/data/estimates2/docs/MigrationStockDocumentation_2015.pdf

19 http://popstats.unhcr.org/en/overview\#_ga=1.234832542.1284217032. 1446475248 
viduals granted complementary forms of protection; or those enjoying temporary protection. Since 2007, the refugee population also includes people in a refugee-like situation.

A metà del 2015 coloro che vivevano in un paese diverso da quello di nascita o di cittadinanza erano, nel mondo, pari a 243.700 .236 unità. Di essi l'8,5 per cento (20,6 milioni) viveva in un paese africano, il 31 per cento ( 75 milioni) in un paese asiatico, un altro 31 per cento (76 milioni) in un paese europeo, il 4 per cento $(9$ milioni) in un paese Latino-americano e il 22 per cento ( 54 milioni) in un paese del Nord America. Rispetto al 1995 lo stock di migranti è cresciuto del 50 per cento ma certamente in misura difforme da un continente all'altro e da un paese all'altro. L'aumento più rilevante si è avuto nell'America del Nord e in Asia (rispettivamente 63 e 61 per cento) e quello più debole nel continente africano ( 26 per cento): tra di due estremi si collocano l'Europa (44 per cento) e l'America Latina (38 per cento).

Volendo osservare più nel dettaglio i singoli paesi, è necessario premettere che, per evitare le distorsioni nel confronto che già si erano notate in merito alle statistiche EUROSTAT, si prenderanno in considerazione quelli ritenuti più significativi tra

a) i paesi che adottano come definizione di "immigrato" (migrant) il criterio della cittadinanza pur trattando separatamente quelli nelle cui stime sono compresi i rifugiati;

b) i paesi che adottano come definizione di "immigrato" (migrant) il criterio della nascita all'estero pur trattando separatamente quelli nelle cui stime sono compresi i rifugiati.

La Tab. 3 presenta la consistenza e le variazioni intervenute negli ultimi 15 anni nello stock di migranti nel primo dei due gruppi di paesi ${ }^{20}$. Si nota che gli incrementi più rilevanti riguardano la Repubblica di Corea (444 per cento) seguita da tre Stati situati nel ricco sud-est della Penisola arabica: Qatar (369 per cento), Oman (196 per cento) e Bahrein (194 per cento). Tali cospicui incrementi hanno portato il Qatar a essere popolato per il 75 per cento da stranieri. In Bahrein gli

20 Sono tutti extraeuropei con le sole eccezioni del Belgio e della Repubblica Ceca. 
stranieri sono il 51 per cento e in Oman sono il 41 per cento della popolazione. Se tra gli immigrati stranieri si includono anche i rifugiati, si evince dalla stessa $T a b .3 b$ che i più forti aumenti interessano, a parte le Maldive, ancora due Stati della penisola arabica: Emirati Arabi Uniti (230 per cento) e Kuwait (154 per cento $)^{21}$ : negli Emirati, gli stranieri costituiscono ben oltre i quattro quinti della popolazione (88 per cento) e nel Kuwait quasi i tre quarti. Meno rilevante è stato l'incremento in Arabia Saudita, che però già nel 2000 contava oltre 5 milioni di stranieri e nel 2015 ne contava oltre 10 milioni configurandosi come lo Stato dove la presenza straniera è la più cospicua in valore assoluto (un terzo della popolazione). I più importanti Stati di origine degli stranieri che vivono nei Paesi del Golfo (primi cinque per ciascun paese) si possono desumere dalla Tab. 4: il primo è sempre l'India, i cui cittadini che in tale area vivono ammontano complessivamente a oltre 8 milioni.

Al di fuori della Penisola Arabica, è la Nigeria che presenta il più elevato tasso di variazione dell'immigrazione straniera ( 145 per cento): i primi cinque paesi di origine sono tutti situati nell'Africa orientale e sono Benin, Ghana, Mali, Togo e Niger. Come si desume da fonte UNHCR, i rifugiati in Nigeria nel 2014 erano 1217 (di cui 531 provenienti dalla Repubblica Democratica del Congo), un sesto di quanti erano nel 2000 (7.270 cui 3194 provenienti dal Chad, 2000 dalla Sierra Leone e quasi 1600 dalla Liberia) ${ }^{22}$. Tra i Paesi che adottano il criterio della cittadinanza solo nello Yemen i rifugiati pesano in misura rilevante sullo stock migratorio (75 per cento) e sono quasi tutti (244.204) somali.

La Tab. 5 presenta la consistenza e le variazioni intervenute negli ultimi 15 anni nello stock di migranti nell'assai più numeroso gruppo di paesi che ne identificano l'origine nel luogo di nascita: data la loro numerosità si è preferito considerare solo quelli che ospitano almeno 500 mila migranti ${ }^{23}$. Da subito si può rilevare che i paesi che, dal 2000 al 2015, hanno sperimentato il più rilevante incremento del flusso immigratorio (se si escludono quelli nelle cui stime sono compresi i

21 Negli Emirati Arabi i rifugiati a fine 2014 erano solo 1.064 e nel Kuwait erano 94.965 (fonte: http://popstats.unhcr.org/en/persons_of_concern; http://www.unhcr.org/ figures-at-a-glance.html)

22 Sempre da fonte UNHCR si desume che nel 2014 gli sfollati interni (internally displaced persons) erano 1.188.000.

23 Si sono esclusi il Sudan e il Sud Sudan che nel luglio 2011 si è reso indipendente dal Sudan. Si è anche esclusa Hong Kong. 
rifugiati) sono europei: Spagna (253 per cento), Italia (173 per cento), Norvegia (154 per cento) e Irlanda (113 per cento). Appartengono all'ex Unione Sovietica anche 4 dei paesi in cui il numero degli immigrati in questi quindici anni si è ridotto: Federazione Russa, Bielorussia, Ucraina, Uzbekistan.

Se si considera la serie che include tra gli immigrati anche i rifugiati, si osserva che i paesi che presentano la più elevata variazione positiva dello stock sono africani, ossia il Chad (393 per cento) e il Sud Africa (214 per cento). E' doveroso sottolineare che il numero di rifugiati nel Chad nel 2014 era pari a 452.877 unità, ossia l'88 per cento dello stock di migranti registrato dall'ONU a metà 2015: tra di essi oltre 357,7 mila provenivano dal Sudan e quasi 92 mila dalla Repubblica Centrafricana. Nel 2000 i rifugiati erano 17,7 mila ed erano quasi tutti sudanesi.

Tra i rimanenti paesi che hanno sperimentato aumenti dello stock di migranti superiori al cento per cento si devono menzionare la Thailandia, il Libano, la Turchia e il Messico: in Libano e in Turchia oltre la metà dello stock è costituito da rifugiati. Di forte peso (oltre il 50 per cento) è ancora la presenza dei rifugiati tra gli immigrati in Etiopia (123.784 dall'Eritrea, 245.097 dalla Somalia, 251791 dal Sud Sudan), in Kenya (424.691 dalla Somalia, 89.226 dal Sud Sudan) e in Uganda (178.217 dalla Repubblica Democratica del Congo, 157.053 dal Sud Sudan). Dei quasi 4 milioni di immigrati in Thailandia la metà (1.978 mila) è nata in Myanmar, in quarto (969 mila) nel Laos e un quinto (805 mila) in Cambogia. Infine si deve sottolineare che 7 su 10 degli immigrati in Messico sono nati negli Stati Uniti d'America. Tra gli immigrati in USA i messicani di nascita, pari a 12 milioni, sono un quarto.

E veniamo, infine, all'Italia che si è vista tra i paesi interessati da uno dei più rilevanti aumenti degli immigrati. Sottolineiamo che in questa sede si considerano immigrati coloro che vivono nel nostro paese e sono nati all'estero e ricordiamo che, diversamente, le statistiche correnti dell'ISTAT si riferiscono agli immigrati stranieri residenti.

Premettiamo innanzitutto che l'ammontare di coloro che sono nati all'estero è nel complesso superiore a quello degli stranieri residenti $(15 \%)$ : non sarebbe da escludere che tale differenza possa essere attribuibile, almeno in parte, a date di riferimento dissimili (il 30 giugno 2015 per i nati all'estero e il 1 gennaio 2015 per gli stranieri) ma, come mostra la $T a b$. 6, se si prendono in considerazione i diversi Stati di provenienza, si percepiscono discordanze così vistose da dover escludere che la mancanza di sincronia nelle rilevazioni possa essere accettata 
come causa esplicativa. Se trascuriamo gli Stati che presentano una numerosità di casi poco rilevante e per i quali le differenze possono essere attribuite a circostanze particolari (si pensi, per esempio, al personale del corpo diplomatico e consolare oppure ai dipendenti di società che ivi hanno sede) come sarebbe ipotizzabile per Emirati Arabi o Kuwait, notiamo per altro verso che per Svizzera, Libia, Lussemburgo il numero di coloro che sono nati in essi e poi immigrati in Italia è 24 volte quello dei loro cittadini che risiedono nel nostro paese e per Canada e Australia è 12 volte. I nati in Argentina, Venezuela e Belgio sono oltre il 700 per cento dei cittadini di quegli Stati e sbalorditivi sono anche i casi di Germania e Francia. Non è superfluo rilevare che tutti i paesi citati, in un passato più o meno recente, sono stati meta della nostra emigrazione, fattore che può ben spiegare l'eccezionale surplus di nati immigrati, parte dei quali cittadini italiani. Vi sono infine una trentina di paesi in cui il numero di coloro che vi sono nati è inferiore a quello dei loro cittadini che risiedono in Italia: non pochi di essi sono quelli che alimentano la nostra attuale immigrazione (Romania innanzitutto, Ucraina, Equador, Filippine, Sri Lanka, Bangladesh, Senegal, Cina). Gli scarti fra le due serie di dati riflettono dunque, almeno in parte, le vicende migratorie che hanno interessato il nostro paese. Esse comunque divergono, e perciò autorizzano a trarre conclusioni differenti in merito agli immigrati in Italia: differenti sia per quanto riguarda la stima del loro ammontare dai singoli paesi di provenienza sia per quanto riguarda la graduatoria, in ordine di importanza, delle provenienze medesime, benché si debba assolutamente sottolineare le non trascurabili eccezioni di Romania, Albania e Marocco, che si trovano a presentare i valori più alti sia di immigrati in essi nati sia di immigrati loro cittadini.

Come concludere queste riflessioni? Come si è iniziato: la difficoltà di definire il fenomeno migratorio è più che mai attuale come lo è quella di pervenire a stime di esso comparabili nel tempo e nello spazio. 


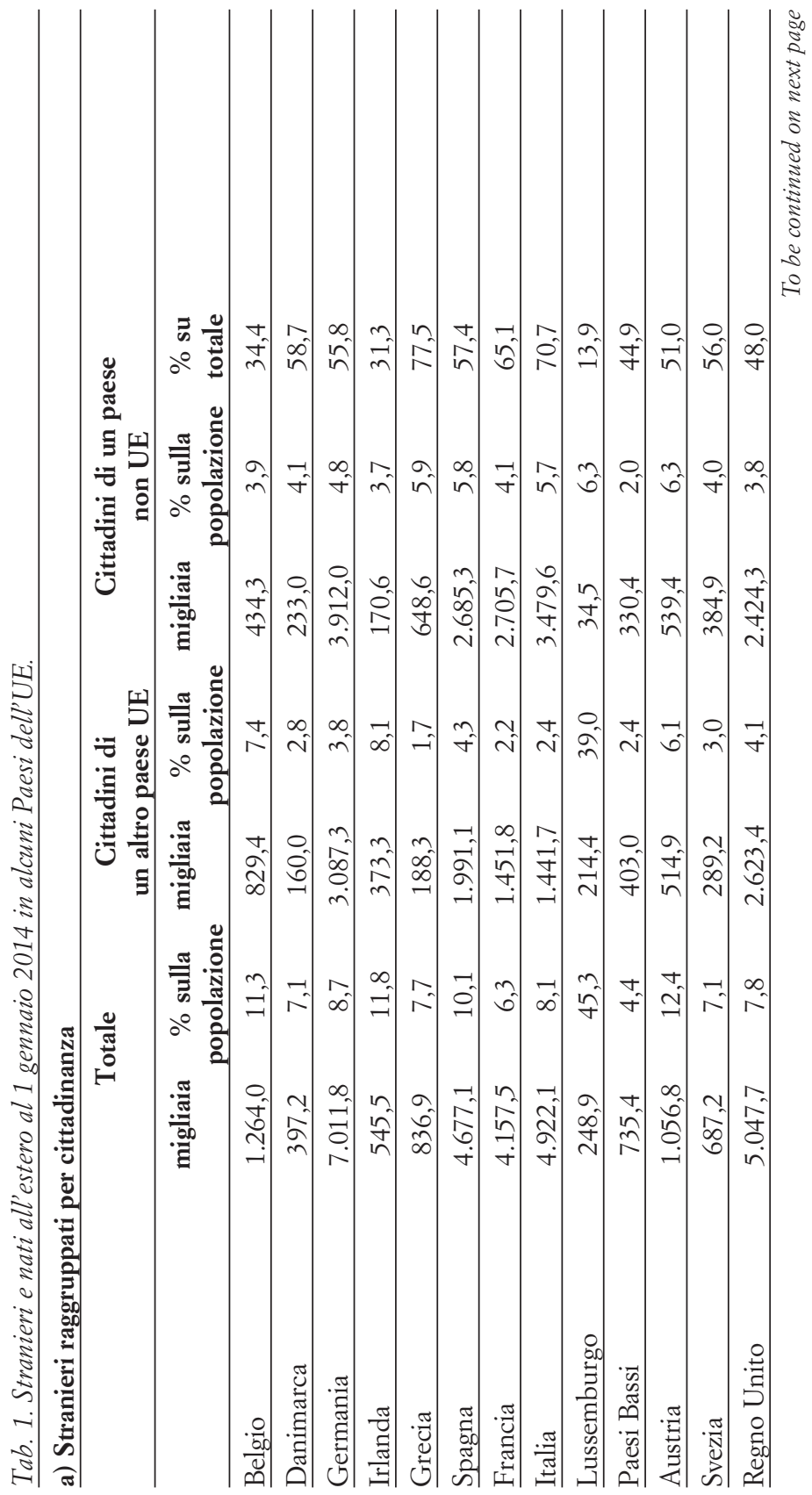




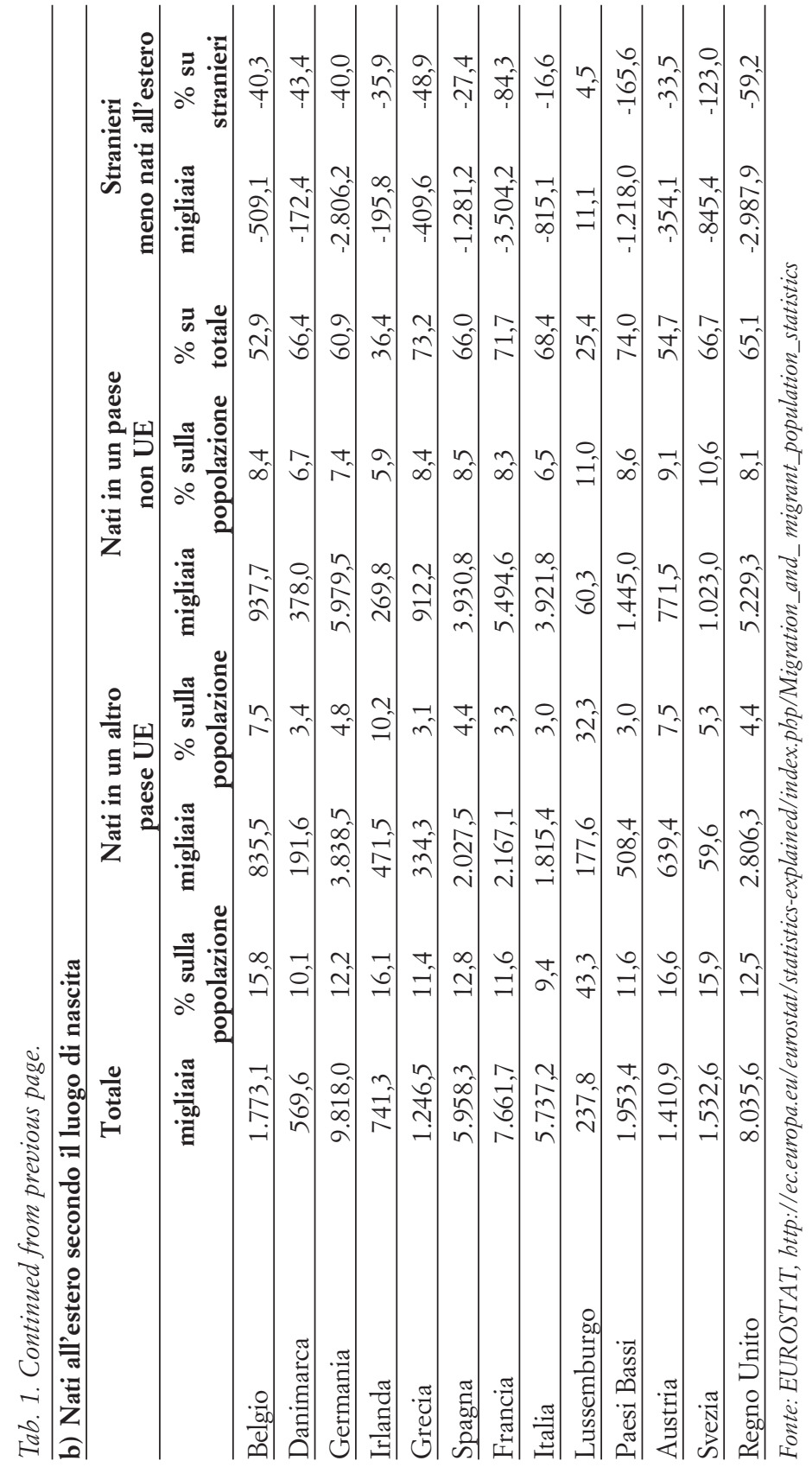




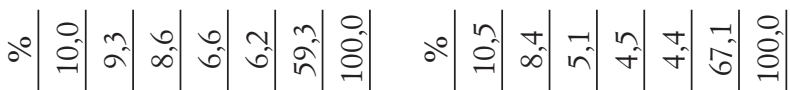

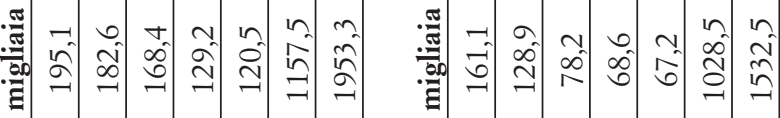

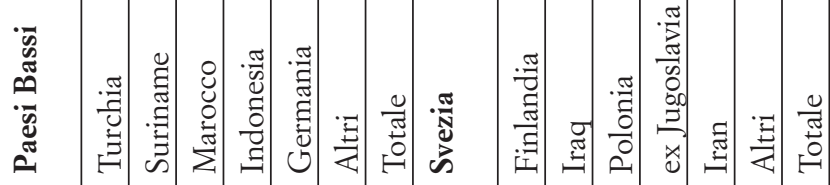

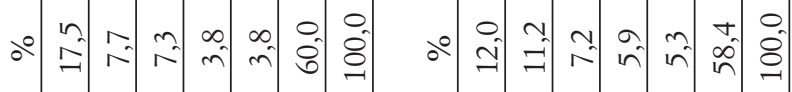

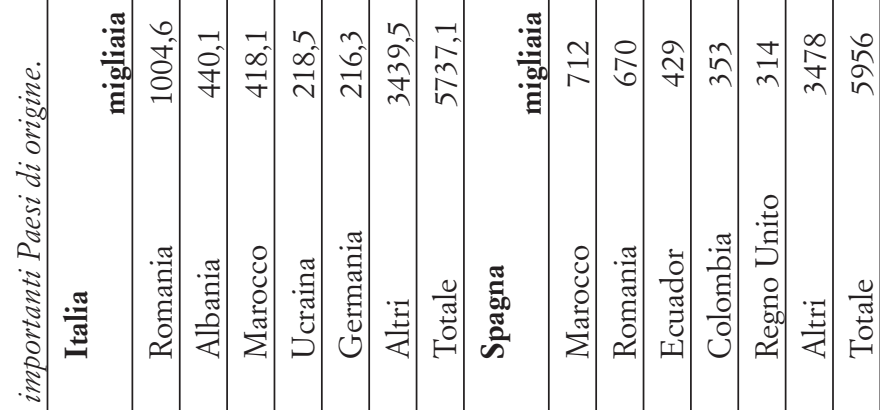

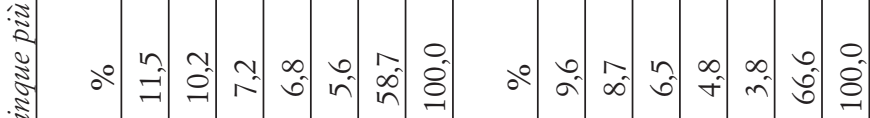

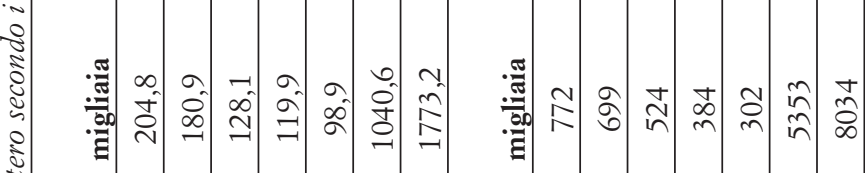

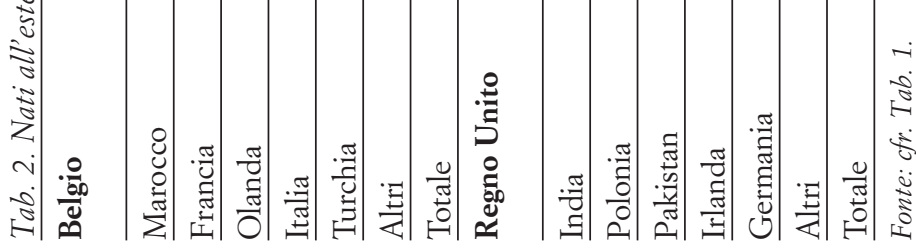


Tab. 3. Stock di migranti nei paesi che adottano il criterio della cittadinanza.

Anni 2000 e 2015.

\begin{tabular}{lccc}
\hline a) Non compresi i rifugiati & $\mathbf{2 0 0 0}$ & $\mathbf{2 0 1 5}$ & Var $\%$ \\
\hline Paesi di destinazione & 244.178 & 1.327 .324 & 443,6 \\
\hline Republic of Korea & 359697 & 1.687 .640 & 369,2 \\
\hline Qatar & 623.608 & 1.844 .978 & 195,9 \\
\hline Oman & 239.361 & 704.137 & 194,2 \\
\hline Bahrain & 57.064 & 160.644 & 181,5 \\
\hline Botswana & 8.206 & 17.620 & 114,7 \\
\hline Mongolia & 508.034 & 978.046 & 92,5 \\
\hline China & 15.543 & 28.585 & 83,9 \\
\hline Mauritius & 220.789 & 405.093 & 83,5 \\
\hline Czech Republic & 27.506 & 46.836 & 70,3 \\
\hline Suriname & 53.034 & 88.511 & 66,9 \\
\hline Morocco & 853.369 & 1.387 .940 & 62,6 \\
\hline Belgium & 36.446 & 56.701 & 55,6 \\
\hline Tunisia & 195.571 & 268.384 & 37,2 \\
\hline Gabon & 23.541 & 32.075 & 36,3 \\
\hline Madagascar & 567.436 & 771.146 & 35,9 \\
\hline Libya & 1.686 .567 & 2.043 .877 & 21,2 \\
\hline Japan & 1.994 .135 & 2.175 .399 & 9,1 \\
\hline Côte d'Ivoire & 98.011 & 73.308 & $-25,2$ \\
\hline Myanmar & 123.529 & 81.598 & $-33,9$ \\
\hline Central African Republic & $\mathbf{2 0 0 0}$ & $\mathbf{2 0 1 5}$ & Var \% \\
\hline b) Compresi i rifugiati & 27.092 & 94.086 & 247,3 \\
\hline Paesi di destinazione & 2.446 .675 & 8.095 .126 & 230,9 \\
\hline Maldives & 1.127 .640 & 2.866 .136 & 154,2 \\
\hline United Arab Emirates & 487.882 & 1.199 .115 & 145,8 \\
\hline Kuwait & 57.366 & 138.162 & 140,8 \\
\hline Nigeria & 143.495 & 344.131 & 139,8 \\
\hline Mauritania & 137.891 & 276.844 & 100,8 \\
\hline Yemen & 1.277 .223 & 2.514 .243 & 96,9 \\
\hline Togo & 5.263 .387 & 10.185 .945 & 93,5 \\
\hline Malaysia & 133.730 & 245.399 & 83,5 \\
\hline Saudi Arabia & 210.525 & 353.881 & 68,1 \\
\hline Benin & 1.927 .845 & 3.112 .026 & 61,4 \\
\hline Iraq & 56.754 & 72.793 & 28,3 \\
\hline Jordan & 832.273 & 875.189 & 5,2 \\
\hline Viet Nam & 250.110 & 242.391 & $-3,1$ \\
\hline Syrian Arab Republic & 211.862 & $-33,4$ \\
\hline Algeria & 228.413 & $-59,2$ \\
\hline Philippines & & & \\
\hline Guinea & & & \\
\hline Font Una & & & \\
\hline
\end{tabular}

Fonte: United Nations, Department of Economic and Social Affairs (2015). Trends in International Migrant Stock: Migrants by Destination and Origin (United Nations database, POP/DB/MIG/ Stock/Rev.2015). 


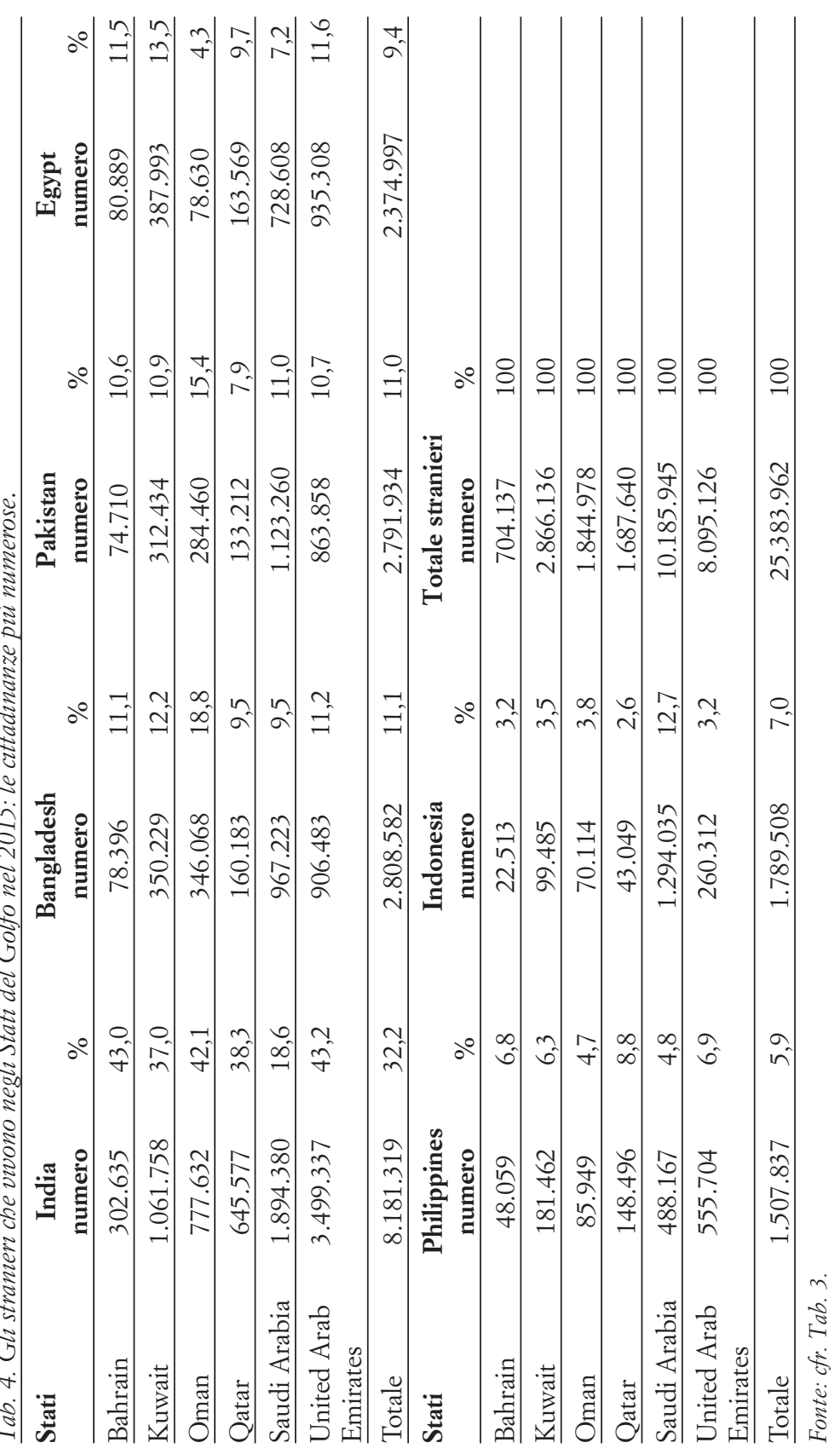


Tab. 5. Stock di migranti nei paesi che adottano il criterio della nascita. Anni 2000 e 2015.

a) Non compresi i rifugiati

Paesi di destinazione

2000

2015

Var \%

Spain

1. 657.285

5. 852.953

253,2

Italy

2. 121.688

5.788. 875

172,8

Norway

292. 440

741.813

153,7

Ireland

350.552

746. 260

112,9

Singapore

1.351 .691

2. 543.638

88,2

United Kingdom of Great Britain 4. 730. 165

8. 543.120

80,6 and Northern Ireland

\begin{tabular}{lccc}
\hline Sweden & 1.003 .798 & 1.639 .771 & 63,4 \\
\hline Switzerland & 1.570 .756 & 2.438 .702 & 55,3 \\
\hline Denmark & 371.026 & 572.520 & 54,3 \\
\hline Australia & 4.386 .250 & 6.763 .663 & 54,2 \\
\hline New Zealand & 678.813 & 1.039 .736 & 53,2 \\
\hline Austria & 996.547 & 1.492 .374 & 49,8 \\
\hline Canada & 5.511 .914 & 7.835 .502 & 42,2 \\
\hline Venezuela (Bolivarian Republic of) & 1.013 .663 & 1.404 .448 & 38,6 \\
\hline Argentina & 1.540 .219 & 2.086 .302 & 35,5 \\
\hline United States of America & 34.814 .053 & 46.627 .102 & 33,9 \\
\hline Germany & 8.992 .631 & 12.005 .690 & 33,5 \\
\hline Portugal & 651.472 & 837.257 & 28,5 \\
\hline Netherlands & 1.556 .337 & 1.979 .486 & 27,2 \\
\hline France & 6.278 .718 & 7.784 .418 & 24,0 \\
\hline Kazakhstan & 2.871 .300 & 3.546 .778 & 23,5 \\
\hline Greece & 1.111 .665 & 1.242 .514 & 11,8 \\
\hline Brazil & 684.596 & 713.568 & 4,2 \\
\hline Russian Federation & 11.900 .297 & 11.643 .276 & $-2,2$ \\
\hline Belarus & 1.123 .586 & 1.082 .905 & $-3,6$ \\
\hline Serbia & 856.763 & 807.441 & $-5,8$ \\
\hline Ukraine & 5.527 .087 & 4.834 .898 & $-12,5$ \\
\hline Uzbekistan & 1.405 .250 & 1.170 .899 & $-16,7$ \\
\hline Poland & 825.251 & 619.403 & $-24,9$ \\
\hline & & & $70607 n t$ \\
\hline
\end{tabular}


Tab. 5. Continued from previous page.

\begin{tabular}{lccc}
\hline $\begin{array}{l}\text { b) Compresi i rifugiati } \\
\text { Paesi di destinazione }\end{array}$ & $\mathbf{2 0 0 0}$ & $\mathbf{2 0 1 5}$ & Var \% \\
\hline Chad & 104.825 & 516.968 & 393,2 \\
\hline South Africa & 1.001 .825 & 3.142 .511 & 213,7 \\
\hline Thailand & 1.257 .821 & 3.913 .258 & 211,1 \\
\hline Lebanon & 692.913 & 1.997 .776 & 188,3 \\
\hline Turkey & 1.280 .963 & 2.964 .916 & 131,5 \\
\hline Mexico & 538.051 & 1.193 .155 & 121,8 \\
\hline Ethiopia & 611.384 & 1.072 .949 & 75,5 \\
\hline Kenya & 699.139 & 1.084 .357 & 55,1 \\
\hline Bangladesh & 987.853 & 1.422 .805 & 44,0 \\
\hline Burkina Faso & 520.039 & 704.676 & 35,5 \\
\hline Uganda & 634.703 & 749.471 & 18,1 \\
\hline Israel & 1.851 .309 & 2.011 .727 & 8,7 \\
\hline Croatia & 585.298 & 576.883 & $-1,4$ \\
\hline Iran (Islamic Republic of) & 2.803 .805 & 2.726 .420 & $-2,8$ \\
\hline Pakistan & 4.181 .912 & 3.628 .956 & $-13,2$ \\
\hline India & 6.411 .272 & 5.240 .960 & $-18,3$ \\
\hline Democratic Republic of the Congo & 744.387 & 545.694 & $-26,7$ \\
\hline Nepal & 717.900 & 518.278 & $-27,8$ \\
\hline Fonte: fr. Tab. & & &
\end{tabular}

Fonte: cfr. Tab. 3. 
Tab. 6. Italia: residenti nati all'estero e residenti stranieri.

\begin{tabular}{|c|c|c|c|}
\hline & $\begin{array}{c}\text { Nati per } \\
\text { Paese di } \\
\text { origine }\end{array}$ & $\begin{array}{l}\text { Stranieri per } \\
\text { Paese di } \\
\text { cittadinanza }\end{array}$ & $\begin{array}{c}\text { Differenza } \\
\%\end{array}$ \\
\hline Totale & 5.788 .875 & 5.014 .437 & 15,4 \\
\hline Afghanistan & 6.568 & 7.654 & $-14,2$ \\
\hline Albania & 447.586 & 490.483 & $-8,7$ \\
\hline Algeria & 22.471 & 22.679 & $-0,9$ \\
\hline Angola & 1.869 & 1.449 & 29,0 \\
\hline Argentina & 73.183 & 8.278 & 784,1 \\
\hline Armenia & 1.256 & 920 & 36,5 \\
\hline Australia & 19.850 & 1.636 & 1113,3 \\
\hline Austria & 16.331 & 6.139 & 166,0 \\
\hline Azerbaijan & 709 & 347 & 104,3 \\
\hline Bangladesh & 97.036 & 115.301 & $-15,8$ \\
\hline Belarus & 32.383 & 8.379 & 286,5 \\
\hline Belgium & 46.393 & 5.421 & 755,8 \\
\hline Benin & 2.566 & 2.505 & 2,4 \\
\hline Bolivia (Plurinational State of) & 15.698 & 14.568 & 7,8 \\
\hline Bosnia and Herzegovina & 11.106 & 29.442 & $-62,3$ \\
\hline$\underline{B r a z i l}$ & 104.207 & 42.587 & 144,7 \\
\hline Bulgaria & 57.691 & 56.576 & 2,0 \\
\hline Burkina Faso & 11.657 & 14.939 & $-22,0$ \\
\hline Burundi & 1.007 & 542 & 85,8 \\
\hline Cabo Verde & 6.160 & 4.621 & 33,3 \\
\hline Cambodia & 1.322 & 208 & 535,6 \\
\hline Cameroon & 11.457 & 12.414 & $-7,7$ \\
\hline Canada & 25.540 & 1.971 & 1195,8 \\
\hline Central African Republic & 238 & 132 & 80,3 \\
\hline Chad & 563 & 494 & 14,0 \\
\hline Chile & 12.427 & 3.364 & 269,4 \\
\hline China & 200.434 & 265.820 & $-24,6$ \\
\hline Colombia & 39.532 & 19.618 & 101,5 \\
\hline Congo & 3.385 & 3.629 & $-6,7$ \\
\hline Costa Rica & 1.508 & 467 & 222,9 \\
\hline Côte d'Ivoire & 25.555 & 25.362 & 0,8 \\
\hline
\end{tabular}


Tab. 6. Continued from previous page.

\begin{tabular}{|c|c|c|c|}
\hline & $\begin{array}{c}\text { Nati per } \\
\text { Paese di } \\
\text { origine } \\
\end{array}$ & $\begin{array}{l}\text { Stranieri per } \\
\text { Paese di } \\
\text { cittadinanza }\end{array}$ & $\begin{array}{c}\text { Differenza } \\
\%\end{array}$ \\
\hline Croatia & 24.709 & 18.259 & 35,3 \\
\hline Cuba & 33.469 & 19.999 & 67,4 \\
\hline Cyprus & 650 & 185 & 251,4 \\
\hline Czech Republic & 10.403 & 5.776 & 80,1 \\
\hline Democratic People's Republic of Korea & 125 & 118 & 5,9 \\
\hline Democratic Republic of the Congo & 6.087 & 3.437 & 77,1 \\
\hline Denmark & 3.238 & 1.953 & 65,8 \\
\hline$\underline{\text { Dominica }}$ & 337 & 1.045 & $-67,8$ \\
\hline Dominican Republic & 42.269 & 28.804 & 46,7 \\
\hline Ecuador & 88.329 & 91.259 & $-3,2$ \\
\hline$\underline{\text { Egypt }}$ & 108.490 & 103.713 & 4,6 \\
\hline El Salvador & 12.464 & 12.981 & $-4,0$ \\
\hline Eritrea & 13.593 & 10.610 & 28,1 \\
\hline Estonia & 1.382 & 1.125 & 22,8 \\
\hline Ethiopia & 30.401 & 8.100 & 275,3 \\
\hline$\underline{\text { Finland }}$ & 2.827 & 1.586 & 78,2 \\
\hline$\underline{\text { France }}$ & 134.403 & 27.696 & 385,3 \\
\hline Gabon & 384 & 240 & 60,0 \\
\hline Gambia & 1.515 & 3.306 & $-54,2$ \\
\hline Georgia & 12.226 & 13.742 & $-11,0$ \\
\hline Germany & 220.013 & 36.749 & 498,7 \\
\hline Ghana & 46.548 & 50.414 & $-7,7$ \\
\hline Greece & 17.405 & 6.585 & 164,3 \\
\hline Guatemala & 2.135 & 737 & 189,7 \\
\hline Guinea & 3.899 & 4.490 & $-13,2$ \\
\hline Guinea-Bissau & 782 & 719 & 8,8 \\
\hline Guyana & 74 & 27 & 174,1 \\
\hline Haiti & 703 & 300 & 134,3 \\
\hline Honduras & 2.323 & 1.785 & 30,1 \\
\hline Hungary & 12.889 & 7.708 & 67,2 \\
\hline Iceland & 207 & 124 & 66,9 \\
\hline India & 136.403 & 147.815 & $-7,7$ \\
\hline
\end{tabular}


Tab. 6. Continued from previous page.

\begin{tabular}{|c|c|c|c|}
\hline & $\begin{array}{c}\text { Nati per } \\
\text { Paese di } \\
\text { origine }\end{array}$ & $\begin{array}{c}\text { Stranieri per } \\
\text { Paese di } \\
\text { cittadinanza }\end{array}$ & $\begin{array}{c}\text { Differenza } \\
\%\end{array}$ \\
\hline$\underline{\text { Indonesia }}$ & 3.159 & 2.416 & 30,8 \\
\hline$\underline{\text { Iran (Islamic Republic of) }}$ & 14.433 & 9.813 & 47,1 \\
\hline$\underline{\text { Iraq }}$ & 3.889 & 2.923 & 33,0 \\
\hline$\underline{\text { Ireland }}$ & 3.629 & 2.598 & 39,7 \\
\hline$\underline{\text { Israel }}$ & 3.883 & 1.989 & 95,2 \\
\hline Jamaica & 346 & 127 & 172,4 \\
\hline Japan & 8.285 & 7.131 & 16,2 \\
\hline Jordan & 2.991 & 1.948 & 53,5 \\
\hline$\underline{\text { Kazakhstan }}$ & 3.851 & 1.547 & 148,9 \\
\hline Kenya & 3.641 & 2.087 & 74,5 \\
\hline$\underline{\text { Kuwait }}$ & 543 & 34 & 1497,1 \\
\hline$\underline{\text { Kyrgyzstan }}$ & 1.326 & 1.193 & 11,1 \\
\hline$\underline{\text { Lao People's Democratic Republic }}$ & 260 & 63 & 312,7 \\
\hline$\underline{\text { Latvia }}$ & 3.200 & 2.689 & 19,0 \\
\hline$\underline{\text { Lebanon }}$ & 7.086 & 3.599 & 96,9 \\
\hline$\underline{\text { Liberia }}$ & 2.006 & 1.562 & 28,4 \\
\hline Libya & 36.222 & 1.697 & 2034,5 \\
\hline$\underline{\text { Lithuania }}$ & 5.646 & 4.875 & 15,8 \\
\hline$\underline{\text { Luxembourg }}$ & 4.799 & 240 & 1899,6 \\
\hline Madagascar & 2.382 & 1.350 & 76,4 \\
\hline Malawi & 171 & 49 & 249,0 \\
\hline$\underline{\text { Malaysia }}$ & 613 & 390 & 57,2 \\
\hline Mali & 4.177 & 6.245 & $-33,1$ \\
\hline Malta & 1.928 & 664 & 190,4 \\
\hline Mauritania & 677 & 770 & $-12,1$ \\
\hline Mauritius & 9.499 & 7.476 & 27,1 \\
\hline Mexico & 8.827 & 4.044 & 118,3 \\
\hline Mongolia & 275 & 163 & 68,7 \\
\hline Montenegro & 2.285 & 2.731 & $-16,3$ \\
\hline Morocco & 425.238 & 449.058 & $-5,3$ \\
\hline Mozambique & 857 & 315 & 172,1 \\
\hline Myanmar & 441 & 301 & 46,5 \\
\hline
\end{tabular}


Tab. 6. Continued from previous page.

\begin{tabular}{|c|c|c|c|}
\hline & $\begin{array}{c}\text { Nati per } \\
\text { Paese di } \\
\text { origine } \\
\end{array}$ & $\begin{array}{l}\text { Stranieri per } \\
\text { Paese di } \\
\text { cittadinanza }\end{array}$ & $\begin{array}{c}\text { Differenza } \\
\%\end{array}$ \\
\hline Nepal & 1.775 & 1.224 & 45,0 \\
\hline Netherlands & 13.034 & 7.849 & 66,1 \\
\hline$\underline{\text { New Zealand }}$ & 901 & 305 & 195,4 \\
\hline Nicaragua & 1.175 & 618 & 90,1 \\
\hline Niger & 1.060 & 1.333 & $-20,5$ \\
\hline Nigeria & 56.259 & 71.158 & $-20,9$ \\
\hline Norway & 1.981 & 1.002 & 97,7 \\
\hline$\underline{\text { Pakistan }}$ & 84.792 & 96.207 & $-11,9$ \\
\hline$\underline{\text { Panama }}$ & 1.021 & 342 & 198,5 \\
\hline Paraguay & 2.636 & 1.749 & 50,7 \\
\hline Peru & 116.038 & 109.668 & 5,8 \\
\hline Philippines & 143.457 & 168.238 & $-14,7$ \\
\hline$\underline{\text { Poland }}$ & 118.641 & 98.694 & 20,2 \\
\hline Portugal & 6.415 & 5.614 & 14,3 \\
\hline Republic of Korea & 3.931 & 3.076 & 27,8 \\
\hline Republic of Moldova & 166.819 & 147.388 & 13,2 \\
\hline$\underline{\text { Romania }}$ & 1.021 .613 & 1.131 .839 & $-9,7$ \\
\hline$\underline{\text { Russian Federation }}$ & 80.750 & 35.211 & 129,3 \\
\hline$\underline{\text { Rwanda }}$ & 778 & 506 & 53,8 \\
\hline Saudi Arabia & 962 & 121 & 695,0 \\
\hline$\underline{\text { Senegal }}$ & 84.815 & 94.030 & $-9,8$ \\
\hline$\underline{\text { Serbia }}$ & 45.581 & 43.811 & 4,0 \\
\hline Seychelles & 978 & 554 & 76,5 \\
\hline Sierra Leone & 1.377 & 1.293 & 6,5 \\
\hline Singapore & 567 & 187 & 203,2 \\
\hline Slovakia & 10.996 & 8.351 & 31,7 \\
\hline Slovenia & 4.699 & 2.574 & 82,6 \\
\hline Somalia & 11.556 & 7.722 & 49,7 \\
\hline South Africa & 5.657 & 594 & 852,4 \\
\hline Spain & 31.829 & 21.286 & 49,5 \\
\hline Sri Lanka & 85.093 & 100.558 & $-15,4$ \\
\hline State of Palestine & 391 & 859 & $-54,5$ \\
\hline
\end{tabular}


Tab. 6. Continued from previous page.

\begin{tabular}{|c|c|c|c|}
\hline & $\begin{array}{c}\text { Nati per } \\
\text { Paese di } \\
\text { origine }\end{array}$ & $\begin{array}{l}\text { Stranieri per } \\
\text { Paese di } \\
\text { cittadinanza }\end{array}$ & $\begin{array}{c}\text { Differenza } \\
\%\end{array}$ \\
\hline Sudan & 2.914 & 2.240 & 30,1 \\
\hline Sweden & 6.411 & 2.968 & 116,0 \\
\hline Switzerland & 198.170 & 8.029 & 2368,2 \\
\hline Syrian Arab Republic & 6.058 & 4.638 & 30,6 \\
\hline Thailand & 9.866 & 5.611 & 75,8 \\
\hline The former Yugoslav Republic of Macedonia & 73.033 & 77.703 & $-6,0$ \\
\hline$\underline{\operatorname{Togo}}$ & 5.152 & 5.163 & $-0,2$ \\
\hline Tunisia & 107.671 & 96.012 & 12,1 \\
\hline Turkey & 20.491 & 19.782 & 3,6 \\
\hline$\underline{\text { Uganda }}$ & 967 & 484 & 99,8 \\
\hline Ukraine & 222.241 & 226.060 & $-1,7$ \\
\hline United Arab Emirates & 370 & 7 & 5185,7 \\
\hline $\begin{array}{l}\text { United Kingdom of Great Britain and } \\
\text { Northern Ireland }\end{array}$ & 64.986 & 25.864 & 151,3 \\
\hline United Republic of Tanzania & 1.604 & 977 & 64,2 \\
\hline United States of America & 54.226 & 14.303 & 279,1 \\
\hline Uruguay & 7.500 & 1.304 & 475,2 \\
\hline Uzbekistan & 2.509 & 1.224 & 105,0 \\
\hline Venezuela (Bolivarian Republic of) & 48.970 & 5.642 & 768,0 \\
\hline$\underline{\text { Viet Nam }}$ & 5.258 & 1.161 & 352,9 \\
\hline Yemen & 263 & 181 & 45,3 \\
\hline Zambia & 874 & 209 & 318,2 \\
\hline Zimbabwe & 881 & 160 & 450,6 \\
\hline
\end{tabular}


